Journal Club

Editor's Note: These short, critical reviews of recent papers in the Journal, written exclusively by graduate students or postdoctoral fellows, are intended to summarize the important findings of the paper and provide additional insight and commentary. For more information on the format and purpose of the Journal Club, please see http://www.jneurosci.org/misc/ifa_features.shtml.

\title{
Elucidating a Core Semantic Network in the Brain-Implications for Disorders of Semantic Cognition
}

\author{
Muireann Irish \\ School of Psychology, the University of New South Wales, Sydney, New South Wales 2052, Australia, Neuroscience Research Australia, Randwick, New \\ South Wales 2031, Australia, and Australian Research Council Centre of Excellence in Cognition and its Disorders, Sydney, New South Wales 2109, \\ Australia \\ Review of Jackson et al.
}

Semantic memory is an individual's store of general conceptual knowledge accumulated and abstracted from previous experiences, without a specific spatial or temporal context. The flexible nature of semantic representations is posited to underlie a host of sophisticated cognitive endeavors, such as language, social cognition, and the capacity to mentally project oneself backward and forward in subjective time (Binder and Desai, 2011; Irish and Piguet, 2013). A finegrained exposition of the neurobiological foundation of the semantic network is required for deconstructing these uniquely human functions and gaining insights into the breakdown of semantic processing in clinical populations.

Despite the centrality of semantic memory for human behavior, understanding of the neural networks specialized for semantic processing has lagged far behind that of episodic memory. This disparity has arisen not only because disproportionate emphasis is placed on understanding the neurocircuitry of episodic memory, but also because of methodological challenges in studying semantic processing. Within the semantic

Received March 27, 2016; revised May 3, 2016; accepted May 5, 2016.

M.I. is supported by Australian Research Council Discovery Early Career Researcher Award DE130100463.

The author declares no competing financial interests.

Correspondence should be addressed to Dr. Muireann Irish, Neurosci-

ence Research Australia, P.0. Box 1165, Randwick, NSW 2031, Australia.

E-mail:m.rish@neura.edu.au.

DOI:10.1523/JNEUROSCI.1005-16.2016

Copyright $\odot 2016$ the authors $\quad 0270-6474 / 16 / 366144-03 \$ 15.00 / 0$ network, the anterior temporal lobe (ATL) is posited to act as a "hub" that integrates modality-specific information to create multimodal representations (Patterson et al., 2007). Significant signal loss and distortion in the ATLs during functional neuroimaging studies, however, has limited our understanding of the connectivity patterns between ATL subregions and the overall functional organization of the semantic network. Therefore, the contribution of the ATLs to semantic processing remains poorly articulated.

A recent study by Jackson et al. (2016) provides insights into the functional organization of the semantic network and conclusive evidence in favor of an ATL-based semantic hub. Using convergent restingstate and active task-based functional neuroimaging methods in healthy young participants, the authors sought to determine the degree of overlap between the functional connectivity of the semantic memory network in the resting state versus that engaged during effortful semantic processing. To circumvent some of the methodological issues associated with studying the ATLs, the authors used a novel dualecho gradient brain EPI technique, which maximizes the signal in the ATLs and maintains high-contrast sensitivity throughout the entire brain. This approach permits comprehensive investigation of connectivity within and between ATL subregions and their respective contributions to semantic cognition, with minimal signal loss. Fur- thermore, the authors captured the functional heterogeneity of the ATLs by subdividing the region into several independently derived semantic regions of interest (ROIs), including the ventral ATL, anterior superior temporal gyrus/superior temporal sulcus (aSTG/STS), and anterior middle temporal gyrus (MTG). The differential connectivity of ATL subregions during resting and active states was then investigated to determine whether a core network subtends multimodal semantic cognition.

The resting-state procedure involved participants lying quietly in the scanner and focusing on a fixation cross. Data were explored using seed-based functional connectivity analyses to compare resting patterns of brain activity in the ATL ROIs to that in all other predetermined ROIs. Correlations between each seed and each semantic ROI were calculated across the group, and an average value for each individual was computed. This statistic, reflecting the strength of connection between the seeds to the predetermined semantic areas, was compared between seeds to determine whether the regions in question formed the same network. In contrast, during the active semantic task, participants were required to make semantic judgments in which they matched a probe word to the most semantically related of two word choices. The degree of semantic relatedness was manipulated according to semantic association and conceptual similarity. Whole-brain psychophysiological interaction analysis was used to determine 
task-specific increases in the relationship between the ventral ATL seed and the a priori semantic network regions during the semantic task. The psychophysiological interaction analyses thus provided the necessary counterpoint to the resting-state functional connectivity data, enabling the authors to explore ATL connectivity during effortful semantic processing.

In line with the authors' predictions, during the resting state, activity in the ventral ATL seed, and to a lesser extent an anterior MTG seed, was highly correlated with a distributed network of regions including the bilateral ATL, inferior frontal gyrus, medial prefrontal cortex, angular gyrus, posterior MTG, medial temporal lobe, and posterior regions, including the bilateral middle and posterior cingulate cortices, precuneus, right precentral and postcentral gyrus, and the cerebellum. Collectively, these regions have been implicated across functional neuroimaging studies of semantic cognition (Binder et al., 2009). In contrast, activity in the aSTG/STS seed overlapped more closely with multiple regions involved in language and auditory processing outside the core semantic network. Thus, the resting-state results extend previous work by demonstrating a clear subdivision of connectivity within the ATL, with ventrolateral aspects occupying a crucial position in the semantic network.

One of the most striking findings of this study concerns the overlap in connectivity during the resting state and effortful semantic processing between the ventral ATL and distinct brain regions implicated in multimodal semantic cognition. Analyses of the psychophysiological interaction effect demonstrated significant connectivity between the ventral ATL and each of the a priori semantic ROIs, underscoring the importance of the ATL as a transmodal hub within a functionally connected semantic network (Patterson et al., 2007). While this overlap was evident, it is important to note that the semantic networks identified using the two approaches were not identical. Psychophysiological interaction analyses revealed large areas of frontal and occipital cortex, which were not evident in the resting-state analyses, a finding the authors interpret as potentially reflecting increased executive control demands of task-based processing.

The study by Jackson et al. (2016) demonstrates a common core semantic network in the brain, the functional connectivity of which is similar regardless of whether the brain is at rest or engaged in effortful semantic processing. The exposition of a defaultsemantic network (Pascual et al., 2015) meshes well with the discovery of a default- mode network that activates when healthy individuals engage in effortful episodicbased tasks and exhibit high levels of resting metabolic activity (Spreng et al., 2009; Andrews-Hanna, 2012). One of the most persuasive arguments for this convergence between effortful episodic processing and the resting state is that, during idle periods, humans invariably engage in an array of spontaneous introspective processes that draw upon episodic memory, such as remembering the past and envisaging the future (Andrews-Hanna, 2012). Importantly, however, the core semantic network overlaps extensively with this default-mode network (Binder and Desai, 2011). In light of recent work emphasizing the necessary interdependence between semantic and episodic memory on both the behavioral (Irish et al., 2012) and neuroanatomical (Burianova et al., 2010) level, it would be interesting to explore the functional coupling between the ventral ATL seed identified here and the default-mode network. This is particularly relevant when we consider that Jackson et al. (2016) found significant functional connection between the ventral ATL and the medial temporal lobe during the resting state. Given recent insights into the putative role of the default-mode network in spontaneous cognition, an experiencesampling approach in which participants report on the content of their thoughts during the resting state scan would further clarify the extent to which internally driven mentation comprises semantic concepts (Binder and Desai, 2011).

A final consideration is how the present findings can inform our understanding of distinct pathophysiological mechanisms underlying disorders of semantic cognition. The neurodegenerative disorder of semantic dementia is of immense interest in this context given that the maximal sites of atrophy in this syndrome reside in the ventrolateral aspects of the ATL (Davies et al., 2009). Patients with semantic dementia show a progressive and transmodal loss of conceptual knowledge manifesting in profound alterations in naming, comprehension, and object recognition. The severity of these deficits correlates robustly with the magnitude of ATL pathology (Mion et al., 2010). Despite the relatively circumscribed nature of ATL atrophy initially seen in semantic dementia, recent studies using task-free resting-state neuroimaging reveal the vulnerability of connectivity between the ATL and a number of functionally relevant processing areas (Guo et al., 2013; La Joie et al., 2014). Collectively, these findings suggest that, in semantic dementia, localized pathology in the ATL gradually spreads via shared nodes to adjacent vulnerable networks in the brain and reinforces the need to consider dynamic interactions within and between large-scale functional networks to appreciate how neuropathological processes propagate in the brain.

In conclusion, the study by Jackson et al. (2016) provides convergent neuroimaging data in support of a core network that subtends multimodal semantic cognition, with preferential connectivity between ventrolateral aspects of the ATLs and the semantic network evident in both active and resting states. This article represents an important advancement in our understanding of the neural architecture of semantic cognition, adding to a growing body of research emphasizing the graded specialization of ATL subregions for the representation of semantic concepts (Rice et al., 2015). Future work within this field will serve to clarify the unique role of the ATLs as a transmodal integrative hub, providing new insights into the foundational role of semantic processing for everyday adaptive functioning, as well as illuminating our understanding of how these processes break down in clinical populations.

\section{References}

Andrews-Hanna JR (2012) The brain's default network and its adaptive role in internal mentation. Neuroscientist 18:251-270. CrossRef Medline

Binder JR, Desai RH (2011) The neurobiology of semantic memory. Trends Cogn Sci 15: 527-536. CrossRef Medline

Binder JR, Desai RH, Graves WW, Conant LL (2009) Where is the semantic system? A critical review and meta-analysis of 120 functional neuroimaging studies. Cereb Cortex 19: 2767-2796. CrossRef Medline

Burianova H, McIntosh AR, Grady CL (2010) A common functional brain network for autobiographical, episodic, and semantic memory retrieval. Neuroimage 49:865-874. CrossRef Medline

Davies RR, Halliday GM, Xuereb JH, Kril JJ, Hodges JR (2009) The neural basis of semantic memory: evidence from semantic dementia. Neurobiol Aging 30:2043-2052. CrossRef Medline

Guo CC, Gorno-Tempini ML, Gesierich B, Henry M, Trujillo A, Shany-Ur T, Jovicich J, Robinson SD, Kramer JH, Rankin KP, Miller BL, Seeley WW (2013) Anterior temporal lobe degeneration produces widespread networkdriven dysfunction. Brain 136:2979-2991. CrossRef Medline

Irish M, Piguet O (2013) The pivotal role of semantic memory in remembering the past and imagining the future. Front Behav Neurosci 7:27. CrossRef Medline

Irish M, Addis DR, Hodges JR, Piguet O 
Considering the role of semantic memory in episodic future thinking: evidence from semantic dementia. Brain 135:2178-2191. CrossRef Medline

Jackson RL, Hoffman P, Pobric G, Lambon Ralph MA (2016) The semantic network at work and rest: differential connectivity of anterior temporal lobe subregions. J Neurosci 36: 1490-1501. CrossRef Medline

La Joie R, Landeau B, Perrotin A, Bejanin A, Egret S, Pélerin A, Mézenge F, Belliard S, de La Sayette V, Eustache F, Desgranges B, Chételat G (2014) Intrinsic connectivity identifies the hippocampus as a main crossroad between Alzheimer's and se- mantic dementia-targeted networks. Neuron 81: 1417-1428. CrossRef Medline

Mion M, Patterson K, Acosta-Cabronero J, Pengas G, Izquierdo-Garcia D, Hong YT, Fryer TD, Williams GB, Hodges JR, Nestor PJ (2010) What the left and right anterior fusiform gyri tell us about semantic memory. Brain 133:3256-3268. CrossRef Medline

Pascual B, Masdeu JC, Hollenbeck M, Makris N, Insausti R, Ding SL, Dickerson BC (2015) Large-scale brain networks of the human left temporal pole: a functional connectivity MRI study. Cereb Cortex 25:680-702. CrossRef Medline

Patterson K, Nestor PJ, Rogers TT (2007) Where do you know what you know? The representation of semantic knowledge in the human brain. Nat Rev Neurosci 8:976-987. CrossRef Medline

Rice GE, Hoffman P, Lambon Ralph MA (2015) Graded specialization within and between the anterior temporal lobes. Ann N Y Acad Sci 1359:84-97. CrossRef Medline

Spreng RN, Mar RA, Kim AS (2009) The common neural basis of autobiographical memory, prospection, navigation, theory of mind, and the default mode: a quantitative meta-analysis. J Cogn Neurosci 21:489510. CrossRef Medline 